\title{
Several Types of Totally Continuous Functions in Double Fuzzy Topological Spaces
}

\author{
Fatimah M. Mohammed, ${ }^{1,2}$ M. S. M. Noorani, ${ }^{1}$ and A. Ghareeb ${ }^{3,4}$ \\ ${ }^{1}$ School of Mathematical Sciences, Universiti Kebangsaan Malaysia, 43600 Bangi, Selangor, Malaysia \\ ${ }^{2}$ College of Education, Tikrit University, Iraq \\ ${ }^{3}$ Department of Mathematics, College of Science in Al-Zulfi, Majmaah University, Al-Zulfi, Saudi Arabia \\ ${ }^{4}$ Department of Mathematics, Faculty of Science, South Valley University, Qena, Egypt
}

Correspondence should be addressed to Fatimah M. Mohammed; fatimah_ukm@yahoo.com

Received 21 March 2014; Accepted 25 June 2014; Published 10 July 2014

Academic Editor: Rustom M. Mamlook

Copyright (C) 2014 Fatimah M. Mohammed et al. This is an open access article distributed under the Creative Commons Attribution License, which permits unrestricted use, distribution, and reproduction in any medium, provided the original work is properly cited.

We introduce the notions of totally continuous functions, totally semicontinuous functions, and semitotally continuous functions in double fuzzy topological spaces. Their characterizations and the relationship with other already known kinds of functions are introduced and discussed.

\section{Introduction}

The concept of fuzzy sets was introduced by Zadeh in his classical paper [1]. In 1968, Chang [2] used fuzzy sets to introduce the notion of fuzzy topological spaces. Çoker [3, 4] defined the intuitionistic fuzzy topological spaces using intuitionistic fuzzy sets. Later on, Demirci and Çoker [5] defined intuitionistic fuzzy topological spaces in KubiakŠostak's sense as a generalization of Chang's fuzzy topological spaces and intuitionistic fuzzy topological spaces. Mondal and Samanta [6] succeeded to make the topology itself intuitionistic. The resulting structure is given the new name "intuitionistic gradation of openness." The name "intuitionistic" did not continue due to some doubts that were thrown about the suitability of this term. These doubts were quickly ended in 2005 by Gutiérrez García and Rodabaugh [7]. They proved that this term is unsuitable in mathematics and applications. Therefore, they replaced the word "intuitionistic" by "double" and renamed its related topologies. The notion of intuitionistic gradation of openness is given the new name "double fuzzy topological spaces."

The fuzzy type of the notion of topology can be studied in the fuzzy mathematics, which has many applications in different branches of mathematics and physics theory. For example, fuzzy topological spaces can be applied in the modeling of spatial objects such as rivers, roads, trees, and buildings. Since double fuzzy topology forms an extension of fuzzy topology and general topology, we think that our results can be applied in modern physics and GIS Problems.

Jain et al. introduced totally continuous, fuzzy totally continuous, and intuitionistic fuzzy totally continuous functions in topological spaces, respectively (see [8-11]).

In this paper, we introduce the notions of totally continuous, totally semicontinuous, and semitotally continuous functions in double fuzzy topological spaces and investigate some of their characterizations. Also, we study the relationships between these new classes and other classes of functions in double fuzzy topological spaces.

\section{Preliminaries}

Throughout this paper, let $X$ be a nonempty set and let $I$ be the closed interval $[0,1], I_{0}=(0,1]$ and $I_{1}=[0,1)$. The set of all fuzzy subsets (resp., fuzzy points) of $X$ is denoted by $I^{X}$ (resp., $P_{t}(X)$ ). For $x \in X$ and $t \in I_{0}$, a fuzzy point is defined by

$$
x_{t}(y)= \begin{cases}t, & \text { if } y=x, \\ 0, & \text { if } y \neq x .\end{cases}
$$


$x_{t} \in \lambda$ if and only if $t \leq \lambda(x)$. We denote a fuzzy set $\lambda$ which is quasicoincident with a fuzzy set $\mu$ by $\lambda q \mu$, if there exists $x \in X$ such that $\lambda(x)+\mu(x)>1$, otherwise, by $\lambda \bar{q} \mu$. Given a function $f: X \rightarrow Y, f(\lambda)$ and $f^{-1}(\lambda)$ are the direct image and the inverse image of $f$, respectively, and are defined by $f(\lambda)(y)=$ $\vee_{f(x)=y} \lambda(x)$ and $f^{-1}(\mu)(x)=\mu(f(x))$ for each $\lambda \in I^{X}, \mu \in I^{Y}$, and $x \in X$, respectively.

Definition 1 (see $[12,13]$ ). The pair of functions $\tau, \tau^{*}: I^{X} \rightarrow$ $I$ is called a double fuzzy topology on $X$ if it satisfies the following conditions.

(O1) $\tau(\lambda) \leq 1-\tau^{*}(\lambda)$ for each $\lambda \in I^{X}$.

(O2) $\tau\left(\lambda_{1} \wedge \lambda_{2}\right) \geq \tau\left(\lambda_{1}\right) \wedge \tau\left(\lambda_{2}\right)$ and $\tau^{*}\left(\lambda_{1} \wedge \lambda_{2}\right) \leq \tau^{*}\left(\lambda_{1}\right) \vee$ $\tau^{*}\left(\lambda_{2}\right)$ for each $\lambda_{1}, \lambda_{2} \in I^{X}$.

(O3) $\tau\left(\vee_{i \in \Gamma}\right) \geq \wedge_{i \in \Gamma} \tau\left(\lambda_{i}\right)$ and $\tau^{*}\left(\vee_{i \in \Gamma}\right) \leq \vee_{i \in \Gamma} \tau^{*}\left(\lambda_{i}\right)$ for any $\left\{\lambda_{i}\right\}_{i \in \Gamma} \subset I^{X}$.

The triplet $\left(X, \tau, \tau^{*}\right)$ is called a double fuzzy topological space (dfts, for short). $\tau(\lambda)$ and $\tau^{*}(\lambda)$ may be interpreted as a gradation of openness and gradation of nonopenness for $\lambda$. A function $f:\left(X, \tau_{1}, \tau_{1}^{*}\right) \rightarrow\left(Y, \tau_{2}, \tau_{2}^{*}\right)$ is said to be a double fuzzy continuous (briefly, dfc) if $\tau_{1}\left(f^{-1}(\mu)\right) \geq \tau_{2}(\mu)$ and $\tau_{1}^{*}\left(f^{-1}(\mu)\right) \leq \tau_{2}^{*}(\mu)$ for each $\mu \in I^{Y}$.

Theorem 2 (see [12-14]). Let $\left(X, \tau, \tau^{*}\right)$ be a dfts. Then, for each $r \in I_{0}, s \in I_{1}$, and $\lambda \in I^{X}$, we define an operator $C_{\tau, \tau^{*}}: I^{X} \times I_{0} \times I_{1} \rightarrow I^{X}$ as follows:

$$
\begin{aligned}
& C_{\tau, \tau^{*}}(\lambda, r, s) \\
& \quad=\bigwedge\left\{\mu \in I^{X} \mid \lambda \leq \mu, \tau(\underline{1}-\mu) \geq r, \tau^{*}(\underline{1}-\mu) \leq s\right\} .
\end{aligned}
$$

For $\lambda, \mu \in I^{X}, r, r_{1}, r_{2} \in I_{0}$, and $s, s_{1}, s_{2} \in I_{1}$, the operator $C_{\tau, \tau^{*}}$ satisfies the following statements.
(C1) $C_{\tau, \tau^{*}}(\underline{0}, r, s)=\underline{0}$.
(C2) $\lambda \leq C_{\tau, \tau^{*}}(\lambda, r, s)$.
(C3) $C_{\tau, \tau^{*}}(\lambda, r, s) \vee C_{\tau, \tau^{*}}(\mu, r, s)=C_{\tau, \tau^{*}}(\lambda \vee \mu, r, s)$.
(C4) $C_{\tau, \tau^{*}}\left(\lambda, r_{1}, s_{1}\right) \leq C_{\tau, \tau^{*}}\left(\lambda, r_{2}, s_{2}\right)$ if $r_{1} \leq r_{2}$ and $s_{1} \geq s_{2}$.
(C5) $C_{\tau, \tau^{*}}\left(C_{\tau, \tau^{*}}(\lambda, r, s), r, s\right)=C_{\tau, \tau^{*}}(\lambda, r, s)$.

Theorem 3 (see [12-14]). Let $\left(X, \tau, \tau^{*}\right)$ be a dfts. Then, for each $r \in I_{0}, s \in I_{1}$, and $\lambda \in I^{X}$, we define an operator $I_{\tau, \tau^{*}}: I^{X} \times I_{1} \times I_{0} \rightarrow I^{X}$ as follows:

$$
I_{\tau, \tau^{*}}(\lambda, r, s)=\bigvee\left\{\mu \in I^{X} \mid \mu \leq \lambda, \tau(\mu) \geq r, \tau^{*}(\mu) \leq s\right\} .
$$

For $\lambda, \mu \in I^{X}, r, r_{1}, r_{2} \in I_{0}$, and $s, s_{1}, s_{2} \in I_{1}$, the operator $I_{\tau, \tau^{*}}$ satisfies the following statements.
(I1) $I_{\tau, \tau^{*}}(\underline{1}-\lambda, r, s)=\underline{1}-C_{\tau, \tau^{*}}(\lambda, r, s)$.
(I2) $I_{\tau, \tau^{*}}(\underline{1}, r, s)=\underline{1}$.
(I3) $I_{\tau, \tau^{*}}(\lambda, r, s) \leq \lambda$.
(I4) $I_{\tau, \tau^{*}}(\lambda, r, s) \wedge I_{\tau, \tau^{*}}(\mu, r, s)=I_{\tau, \tau^{*}}(\lambda \wedge \mu, r, s)$.

(I5) $I_{\tau, \tau^{*}}\left(\lambda, r_{1}, s_{1}\right) \geq I_{\tau, \tau^{*}}\left(\lambda, r_{2}, s_{2}\right)$ if $r_{1} \leq r_{2}$ and $s_{1} \geq s_{2}$.

(I6) $I_{\tau, \tau^{*}}\left(I_{\tau, \tau^{*}}(\lambda, r, s), r, s\right)=I_{\tau, \tau^{*}}(\lambda, r, s)$.

(I7) If $I_{\tau, \tau^{*}}\left(C_{\tau, \tau^{*}}(\lambda, r, s), r, s\right)=\lambda$, then $C_{\tau, \tau^{*}}\left(I_{\tau, \tau^{*}}(\underline{1}-\right.$ $\lambda, r, s), r, s)=\underline{1}-\lambda$.

Definition 4 (see $[12,15])$. Let $\left(X, \tau, \tau^{*}\right)$ be a dfts, $\lambda \in I^{X}, r \in$ $I_{0}$, and $s \in I_{1}$. A fuzzy set $\lambda$ is called:

(1) an $(r, s)$-fuzzy semiopen (for short, $(r, s)$-fso) if $\lambda \leq$ $C_{\tau, \tau^{*}}\left(I_{\tau, \tau^{*}}(\lambda, r, s), r, s\right)$. A fuzzy set $\lambda$ is called $(r, s)$ fuzzy semiclosed (for short, $(r, s)$-fsc) if $\underline{1}-\lambda$ is an $(r, s)$-fuzzy semiopen set,

(2) an $(r, s)$-fuzzy preopen (for short, $(r, s)$-fpo) if $\lambda \leq$ $I_{\tau, \tau^{*}}\left(C_{\tau, \tau^{*}}(\lambda, r, s), r, s\right)$. A fuzzy set $\lambda$ is called $(r, s)$ fuzzy preclosed (for short, $(r, s)$-fpc) if $\underline{1}-\lambda$ is an $(r, s)$ fuzzy preopen set.

Definition 5 (see [16]). Let $\left(X, \tau, \tau^{*}\right)$ be a dfts, $\lambda \in I^{X}, x_{t} \in$ $P_{t}(X), r \in I_{0}$, and $s \in I_{1}$. A fuzzy set $\lambda$ is called $(r, s)$-fuzzy Q-neighborhood of $x_{t}$, if $\tau(\lambda) \geq r, \tau^{*}(\lambda) \leq s$, and $x_{t} q \lambda$.

Definition 6. Let $f:\left(X, \tau_{1}, \tau_{1}^{*}\right) \rightarrow\left(Y, \tau_{2}, \tau_{2}^{*}\right)$ be a function between dfts's $\left(X, \tau_{1}, \tau_{1}^{*}\right)$ and $\left(Y, \tau_{2}, \tau_{2}^{*}\right)$. Then $f$ is called:

(1) double fuzzy open [15], if $\tau_{2}(f(\lambda)) \geq \tau_{1}(\lambda)$ and $\tau_{2}^{*}(f(\lambda)) \leq \tau_{1}^{*}(\lambda)$, for each $\lambda \in I^{X}, r \in I_{0}$, and $s \in I_{1} ;$

(2) double fuzzy closed [6] if $\tau_{2}(\underline{1}-f(\lambda)) \geq \tau_{1}(\underline{1}-\lambda)$ and $\tau_{2}^{*}(\underline{1}-f(\lambda)) \leq \tau_{1}^{*}(\underline{1}-\lambda)$, for each $\lambda \in I^{X}, r \in I_{0}$, and $s \in I_{1}$.

Definition 7 (see [15]). Let $\left(X, \tau, \tau^{*}\right)$ be a dfts, $r \in I_{0}$, and $s \in I_{1}$. The two fuzzy sets $\lambda, \mu \in I^{X}$ are said to be $(r, s)$-fuzzy separated if and only if $\lambda \bar{q} C_{\tau, \tau^{*}}(\mu, r, s)$ and $\mu \bar{q} C_{\tau, \tau^{*}}(\lambda, r, s)$. A fuzzy set which cannot be expressed as a union of any two $(r, s)$-fuzzy separated sets is said to be $(r, s)$-fuzzy connected.

\section{Totally Continuous and Totally Semicontinuous Functions in Double Fuzzy Topological Spaces}

In this section, some new classes of functions are introduced. Their characterizations and relationship with other functions are introduced.

Definition 8. Let $\left(X, \tau, \tau^{*}\right)$ be a dfts, $\lambda \in I^{X}, r \in I_{0}$, and $s \in I_{1}$. A fuzzy set $\lambda$ is called:

(1) an $(r, s)$-fuzzy semiclopen (for short, $(r, s)$-fsco) if $\lambda$ is an $(r, s)$-fso set and $(r, s)$-fsc set;

(2) an $(r, s)$-fuzzy preclopen (for short, $(r, s)$-fpco) if $\lambda$ is an $(r, s)$-fpo set and $(r, s)$-fpc set.

Definition 9. Let $f:\left(X, \tau_{1}, \tau_{1}^{*}\right) \rightarrow\left(Y, \tau_{2}, \tau_{2}^{*}\right)$ be a function between dfts's $\left(X, \tau_{1}, \tau_{1}^{*}\right)$ and $\left(Y, \tau_{2}, \tau_{2}^{*}\right)$. Then $f$ is called:

(1) double fuzzy totally continuous (briefly, dftc) if $f^{-1}(\mu)$ is $(r, s)$-fco, for each $\mu \in I^{Y}, r \in I_{0}$, and $s \in I_{1}$ such that $\tau_{2}(\mu) \geq r$ and $\tau_{2}^{*}(\mu) \leq s$, 
(2) double fuzzy totally semicontinuous (briefly, dftsc) if $f^{-1}(\mu)$ is $(r, s)$-fsco, for each $\mu \in I^{Y}, r \in I_{0}$, and $s \in I_{1}$ such that $\tau_{2}(\mu) \geq r$ and $\tau_{2}^{*}(\mu) \leq s$,

(3) double fuzzy totally precontinuous (briefly, $\mathrm{dftpc}$ ) if $f^{-1}(\mu)$ is $(r, s)$-fpco, for each $\mu \in I^{Y}, r \in I_{0}$, and $s \in I_{1}$ such that $\tau_{2}(\mu) \geq r$ and $\tau_{2}^{*}(\mu) \leq s$,

(4) double fuzzy semicontinuous (briefly, dfsc) if $f^{-1}(\mu)$ is $(r, s)$-fso, for each $\mu \in I^{Y}, r \in I_{0}$, and $s \in I_{1}$ such that $\tau_{2}(\mu) \geq r$ and $\tau_{2}^{*}(\mu) \leq s$,

(5) double fuzzy semiopen if $f(\lambda)$ is an $(r, s)$-fso in $I^{Y}$, for each $(r, s)$-fo set $\lambda \in I^{X}, r \in I_{0}$, and $s \in I_{1}$,

(6) double fuzzy semiclosed if $f(\lambda)$ is an $(r, s)$-fsc in $I^{Y}$, for each $(r, s)$-fc set $\lambda \in I^{X}, r \in I_{0}$, and $s \in I_{1}$.

Remark 10. A fuzzy set $\lambda$ in a dfts $\left(X, \tau, \tau^{*}\right)$ is $(r, s)$-fco if and only if it is an $(r, s)$-fsco and $(r, s)$-fpco set, where $r \in I_{0}$ and $s \in I_{1}$.

Theorem 11. Let $f:\left(X, \tau_{1}, \tau_{1}^{*}\right) \rightarrow\left(Y, \tau_{2}, \tau_{2}^{*}\right)$ be a function. Then the following are equivalent:

(1) $f$ is a dftc function,

(2) $f^{-1}(\mu)$ is an $(r, s)$-fco set of $I^{X}$ for each $\mu \in I^{Y}, r \in I_{0}$, and $s \in I_{1}$ such that $\tau_{2}(\underline{1}-\mu) \geq r$ and $\tau_{2}^{*}(\underline{1}-\mu) \leq s$,

(3) $C_{\tau_{1}, \tau_{1}^{*}}\left(f^{-1}(\mu), r, s\right) \leq f^{-1}\left(C_{\tau_{2}, \tau_{2}^{*}}(\mu, r, s)\right)$ and $f^{-1}(\mu) \leq$ $I_{\tau_{1}, \tau_{1}^{*}}\left(f^{-1}\left(C_{\tau_{2}, \tau_{2}^{*}}(\mu, r, s)\right), r, s\right)$ for each $\mu \in I^{Y}, r \in I_{0}$, and $s \in I_{1}$

(4) $f^{-1}\left(I_{\tau_{2}, \tau_{2}^{*}}(\mu, r, s)\right) \leq I_{\tau_{1}, \tau_{1}^{*}}\left(f^{-1}(\mu), r, s\right)$ and $C_{\tau_{1}, \tau_{1}^{*}}\left(f^{-1}\left(I_{\tau_{1}, \tau_{1}^{*}}(\mu, r, s)\right), r, s\right) \leq f^{-1}(\mu)$ for each $\mu \in I^{Y}, r \in I_{0}$, and $s \in I_{1}$.

Proof. (1) $\Rightarrow(2)$ : Let $\tau_{2}(\underline{1}-\mu) \geq r$ and $\tau_{2}^{*}(\underline{1}-\mu) \leq s$. By using (1), $f^{-1}(\underline{1}-\mu)$ is an $(r, s)$-fco set in $I^{X}$. Since

$$
\begin{aligned}
\tau_{1}\left(\underline{1}-f^{-1}(\mu)\right)= & \tau_{1}\left(f^{-1}(\underline{1}-\mu)\right) \geq r, \\
\tau_{1}^{*}\left(\underline{1}-f^{-1}(\mu)\right)= & \tau_{1}^{*}\left(f^{-1}(\underline{1}-\mu)\right) \leq s, \\
\tau_{1}\left(\underline{1}-\left(\underline{1}-f^{-1}(\mu)\right)\right) & =\tau_{1}\left(f^{-1}(\underline{1}-(\underline{1}-\mu))\right) \\
& =\tau_{1}\left(f^{-1}(\mu)\right) \geq r, \\
\tau_{1}^{*}\left(\underline{1}-\left(\underline{1}-f^{-1}(\mu)\right)\right) & =\tau_{1}^{*}\left(f^{-1}(\underline{1}-(\underline{1}-\mu))\right) \\
& =\tau_{1}^{*}\left(f^{-1}(\mu)\right) \leq s,
\end{aligned}
$$

thus $f^{-1}(\mu)$ is an $(r, s)$-fco set in $I^{X}$.

(2) $\Rightarrow(3)$ : Let $\mu \in I^{Y}$. Then, $C_{\tau_{2}, \tau_{2}^{*}}(\mu, r, s)$ is $(r, s)$-fc set in $I^{Y}$. By $(2), f^{-1}\left(C_{\tau_{2}, \tau_{2}^{*}}(\mu, r, s)\right)$ is $(r, s)$-fco in $I^{X}$. Hence,

$$
\begin{aligned}
C_{\tau_{1}, \tau_{1}^{*}}\left(f^{-1}(\mu), r, s\right) & \leq C_{\tau_{1}, \tau_{1}^{*}}\left(f^{-1}\left(C_{\tau_{2}, \tau_{2}^{*}}(\mu, r, s)\right), r, s\right) \\
& =f^{-1}\left(C_{\tau_{2}, \tau_{2}^{*}}(\mu, r, s)\right) .
\end{aligned}
$$

Again by using (2),

$$
\begin{aligned}
f^{-1}(\mu) & \leq f^{-1}\left(C_{\tau_{2}, \tau_{2}^{*}}(\mu, r, s)\right) \\
& =I_{\tau_{1}, \tau_{1}^{*}}\left(f^{-1}\left(C_{\tau_{2}, \tau_{2}^{*}}(\mu, r, s)\right)\right) .
\end{aligned}
$$

(3) $\Rightarrow$ (4): Let $\mu \in I^{Y}$. By using (3), we have

$$
\begin{aligned}
f^{-1}\left(C_{\tau_{2}, \tau_{2}^{*}}(\underline{1}-\mu, r, s)\right) & \geq C_{\tau_{1}, \tau_{1}^{*}}\left(f^{-1}(\underline{1}-\mu), r, s\right) \\
& =C_{\tau_{1}, \tau_{1}^{*}}\left(\underline{1}-f^{-1}(\mu), r, s\right) .
\end{aligned}
$$

Hence,

$$
\begin{aligned}
f^{-1}\left(I_{\tau_{2}, \tau_{2}^{*}}(\mu, r, s)\right) & =f^{-1}\left(\underline{1}-\left(C_{\tau_{2}, \tau_{2}^{*}}(\underline{1}-\mu, r, s)\right)\right) \\
& =\underline{1}-f^{-1}\left(C_{\tau_{2}, \tau_{2}^{*}}(\underline{1}-\mu, r, s)\right) \\
& \leq \underline{1}-\left(C_{\tau_{1}, \tau_{1}^{*}}\left(\underline{1}-f^{-1}(\mu)\right), r, s\right) \\
& =I_{\tau_{1}, \tau_{1}^{*}}\left(f^{-1}(\mu), r, s\right) .
\end{aligned}
$$

By using (3), we have

$$
\begin{aligned}
f^{-1} & \left(I_{\tau_{2}, \tau_{2}^{*}}(\underline{1}-\mu, r, s)\right) \\
& \leq I_{\tau_{1}, \tau_{1}^{*}}\left(f^{-1}\left(C_{\tau_{2}, \tau_{2}^{*}}(\underline{1}-\mu, r, s)\right), r, s\right) \\
& =I_{\tau_{1}, \tau_{1}^{*}}\left(f^{-1}\left(\underline{1}-\left(I_{\tau_{2}, \tau_{2}^{*}}(\mu, r, s)\right), r, s\right)\right) \\
& =I_{\tau_{1}, \tau_{1}^{*}}\left(\underline{1}-\left(f^{-1}\left(I_{\tau_{1}, \tau_{1}^{*}}(\mu, r, s)\right), r, s\right)\right) .
\end{aligned}
$$

Hence,

$$
\begin{aligned}
f^{-1}(\mu) & =\underline{1}-f^{-1}(\underline{1}-\mu) \\
& \geq \underline{1}-\left(I_{\tau_{1}, \tau_{1}^{*}}\left(\underline{1}-f^{-1}\left(I_{\tau_{2}, \tau_{2}^{*}}(\mu, r, s), r, s\right)\right)\right) \\
& =C_{\tau_{1}, \tau_{1}^{*}}\left(f^{-1}\left(I_{\tau_{2}, \tau_{2}^{*}}(\mu, r, s)\right), r, s\right) .
\end{aligned}
$$

(4) $\Rightarrow$ (1): Let $\mu \in I^{Y}$ such that $\mu=I_{\tau_{2}, \tau_{2}^{*}}(\mu, r, s)$. By using (4), we have

$$
f^{-1}(\mu)=f^{-1}\left(I_{\tau_{2}, \tau_{2}^{*}}(\mu, r, s)\right) \leq I_{\tau_{1}, \tau_{1}^{*}}\left(f^{-1}(\mu), r, s\right) .
$$

Hence, $f^{-1}(\mu)=I_{\tau_{2}, \tau_{2}^{*}}\left(f^{-1}(\mu), r, s\right)$; that is, $\tau_{1}\left(f^{-1}(\mu)\right) \geq r$ and $\tau_{1}^{*}\left(f^{-1}(\mu)\right) \leq s$. By using (4), we have

$$
\begin{aligned}
f^{-1}(\mu) & \geq C_{\tau_{1}, \tau_{1}^{*}}\left(f^{-1}\left(I_{\tau_{2}, \tau_{2}^{*}}(\mu, r, s)\right), r, s\right) \\
& =C_{\tau_{1}, \tau_{1}^{*}}\left(f^{-1}(\mu), r, s\right) .
\end{aligned}
$$

Hence, $f^{-1}(\mu)=\left(C_{\tau_{1}, \tau_{1}^{*}}\left(f^{-1}(\mu), r, s\right)\right)$; that is, $\tau_{1}\left(\underline{1}-f^{-1}(\mu)\right) \geq$ $r$ and $\tau_{1}^{*}\left(\underline{1}-f^{-1}(\mu)\right) \leq s$. Therefore $f^{-1}(\mu)$ is an $(r, s)$-fco set in $I^{X}$. Thus, $f$ is dftc function. 
Definition 12. Let $\left(X, \tau, \tau^{*}\right)$ be a dfts. Then, it is called:

(1) double fuzzy semiregular (resp., double fuzzy clopen regular) if, for each $(r, s)$-fsc (resp., $(r, s)$-fco) set $v$ of $I^{x}, r \in I_{0}, s \in I_{1}$ and each fuzzy point $x_{t} \notin \nu$, there exist disjoint $(r, s)$-fso (resp., $(r, s)$-fo) sets $\lambda$ and $\mu$ such that $\nu \leq \lambda$ and $x_{t} \in \mu$,

(2) double fuzzy s-regular (resp., double fuzzy ultraregular) if, for each $(r, s)$-fc set $v$ of $I^{X}$ and each $x_{t} \notin v$, $r \in I_{0}$, and $s \in I_{1}$, there exist disjoint $(r, s)$-fso (resp., $(r, s)$-fco) sets $\lambda$ and $\mu$ such that $\nu \leq \lambda$ and $x_{t} \in \mu$,

(3) double fuzzy s-normal if, for each pair of nonzero disjoint $(r, s)$-fc sets can be separated by disjoint $(r, s)$ fso sets, $r \in I_{0}$ and $s \in I_{1}$,

(4) double fuzzy clopen normal if, for each pair of disjoint $(r, s)$-fco sets $\lambda$ and $\mu$ of $I^{X}$, there exist two disjoint $(r, s)$-fo sets $\alpha$ and $\beta, r \in I_{0}$ and $s \in I_{1}$ such that $\lambda \leq \alpha$ and $\mu \leq \beta$.

Theorem 13. If $f:\left(X, \tau_{1}, \tau_{1}^{*}\right) \rightarrow\left(Y, \tau_{2}, \tau_{2}^{*}\right)$ is dftc injective semiopen function from a double fuzzy clopen regular space $\left(X, \tau_{1}, \tau_{1}^{*}\right)$ onto a double fuzzy space $\left(Y, \tau_{2}, \tau_{2}^{*}\right)$, then $\left(Y, \tau_{2}, \tau_{2}^{*}\right)$ is double fuzzy s-regular.

Proof. Suppose $\beta$ is an $(r, s)$-fc set in $I^{Y}, y \notin \beta$, and $f$ is dftc; $f^{-1}(\beta)$ is $(r, s)$-fco set in $I^{X}$, for each $\beta \in I^{Y}, r \in I_{0}$, and $s \in I_{1}$ such that $\tau_{2}(\beta) \geq r$ and $\tau_{2}^{*}(\beta) \leq s$. Put $y=f\left(x_{t}\right)$ and $\alpha=f^{-1}(\beta)$; then $x_{t} \notin \alpha$. Since $\left(X, \tau_{1}, \tau_{1}^{*}\right)$ is double fuzzy clopen regular, then there exist disjoint $(r, s)$-fo sets $\lambda$ and $\mu$ such that $\alpha \leq \lambda$ and $x_{t} \in \mu$. This implies

$$
\begin{aligned}
& \beta=f(\alpha) \leq f(\lambda), \\
& y=f\left(x_{t}\right) \leq f(\mu) .
\end{aligned}
$$

But $f$ is injective and double fuzzy semiopen, so

$$
f(\lambda) \wedge f(\mu)=f(\lambda \wedge \mu)=f(\underline{0})=\underline{0},
$$

and $f(\lambda)$ and $f(\mu)$ are $(r, s)$-fso sets in $I^{Y}, r \in I_{0}$, and $s \in I_{1}$. Therefore $\left(Y, \tau_{2}, \tau_{2}^{*}\right)$ is double fuzzy s-regular.

Theorem 14. If $f:\left(X, \tau_{1}, \tau_{1}^{*}\right) \rightarrow\left(Y, \tau_{2}, \tau_{2}^{*}\right)$ is dftc injective semiopen function from a double fuzzy clopen normal space $\left(X, \tau_{1}, \tau_{1}^{*}\right)$ onto a double fuzzy space $\left(Y, \tau_{2}, \tau_{2}^{*}\right)$, then $\left(Y, \tau_{2}, \tau_{2}^{*}\right)$ is double fuzzy s-normal.

Proof. Suppose $\alpha$ and $\beta$ are any two disjoint $(r, s)$-fc sets in $I^{Y}$, $r \in I_{0}$, and $s \in I_{1}$. Since $f$ is dftc, $f^{-1}(\alpha)$ and $f^{-1}(\beta)$ are $(r, s)$ fco subsets of $I^{X}$, for each $\alpha$ and $\beta \in I^{Y}, r \in I_{0}$, and $s \in I_{1}$ such that $\tau_{2}(\alpha) \geq r$ and $\tau_{2}^{*}(\alpha) \leq s, \tau_{2}(\beta) \geq r$, and $\tau_{2}^{*}(\beta) \leq s$. Put $\lambda=f^{-1}(\alpha)$ and $\mu=f^{-1}(\beta)$ and $f$ is injective, so

$$
\lambda \wedge \mu=f^{-1}(\alpha) \wedge f^{-1}(\beta)=f^{-1}(\alpha \wedge \beta)=f^{-1}(\underline{0})=\underline{0} .
$$

Now, $\left(X, \tau_{1}, \tau_{1}^{*}\right)$ is double fuzzy clopen normal; then there exist disjoint $(r, s)$-fo sets $\delta_{1}$ and $\delta_{2}, r \in I_{0}$ and $s \in I_{1}$ such that $\lambda \leq \delta_{1}$ and $\mu \leq \delta_{2}$ which implies $\alpha=f(\lambda) \leq f\left(\delta_{1}\right)$ and $\beta=f(\mu) \leq f\left(\delta_{2}\right)$, also by the hypothesis of $f$ being injective double fuzzy semiopen we have $f\left(\delta_{1}\right)$ and $f\left(\delta_{2}\right)$ which are disjoint $(r, s)$-fso sets. Therefore $\left(Y, \tau_{2}, \tau_{2}^{*}\right)$ is double fuzzy snormal.

Theorem 15. Let $f:\left(X, \tau_{1}, \tau_{1}^{*}\right) \rightarrow\left(Y, \tau_{2}, \tau_{2}^{*}\right)$ be dftc, closed injective function. If $\left(Y, \tau_{2}, \tau_{2}^{*}\right)$ is double fuzzy s-regular, then $\left(X, \tau_{1}, \tau_{1}^{*}\right)$ is double fuzzy ultraregular.

Proof. Suppose $\beta$ is an $(r, s)$-fc set not containing $x_{t}, r \in I_{0}$, $s \in I_{1}$ and $f$ is double fuzzy closed function, so $\tau_{2}(\underline{1}-f(\beta)) \geq$ $\tau_{1}(\underline{1}-\beta)$ and $\tau_{2}^{*}(\underline{1}-f(\beta)) \leq \tau_{1}^{*}(\underline{1}-\beta)$, for each $\beta \in I^{X}, r \in I_{0}$, and $s \in I_{1}$ such that $f(\beta)$ is $(r, s)$-fc set in $I^{Y}$ not containing $f\left(x_{t}\right)$. But $\left(Y, \tau_{2}, \tau_{2}^{*}\right)$ is double fuzzy s-regular; then there exist disjoint $(r, s)$-fso sets $\lambda$ and $\mu$ such that $f\left(x_{t}\right) \leq \lambda$ and $f(\beta) \leq$ $\mu$. This implies

$$
\begin{aligned}
& x_{t} \in f^{-1}(\lambda), \\
& \beta \leq f^{-1}(\mu) .
\end{aligned}
$$

But $f$ is dftc, so $f^{-1}(\lambda)$ and $f^{-1}(\mu)$ are $(r, s)$-fco, for each $\lambda$ and $\mu \in I^{Y}, r \in I_{0}$, and $s \in I_{1}$ such that $\tau_{2}(\lambda) \geq r, \tau_{2}^{*}(\lambda) \leq s$, $\tau_{2}(\mu) \geq r$, and $\tau_{2}^{*}(\mu) \leq s$ such that $f^{-1}(\lambda)$ and $f^{-1}(\mu)$ are $(r, s)$-fco sets in $I^{X}$. Also $f$ is injective; then

$$
f(\lambda) \wedge f(\mu)=f(\lambda \wedge \mu)=f(\underline{0})=\underline{0} .
$$

Therefore, $\left(X, \tau_{1}, \tau_{1}^{*}\right)$ is double fuzzy ultraregular.

Theorem 16. Let $f:\left(X, \tau_{1}, \tau_{1}^{*}\right) \rightarrow\left(Y, \tau_{2}, \tau_{2}^{*}\right)$ be a function. Then the following are equivalent:

(1) $f$ is a dftsc function,

(2) $f^{-1}(\mu)$ is an $(r, s)$-fco set of $I^{X}$ for each $\mu \in I^{Y}, r \in I_{0}$, and $s \in I_{1}$ such that $\tau_{2}(\underline{1}-\mu) \geq r$ and $\tau_{2}^{*}(\underline{1}-\mu) \leq s$,

(3) $C_{\tau_{1}, \tau_{1}^{*}}\left(f^{-1}(\mu), r, s\right) \leq f^{-1}\left(C_{\tau_{2}, \tau_{2}^{*}}(\mu, r, s)\right)$ and $f^{-1}(\mu) \leq$ $I_{\tau_{1}, \tau_{1}^{*}}\left(f^{-1}\left(C_{\tau_{2}, \tau_{2}^{*}}(\mu, r, s)\right), r, s\right)$ for each $\mu \in I^{Y}, r \in I_{0}$, and $s \in I_{1}$

(4) $f^{-1}\left(I_{\tau_{2}, \tau_{2}^{*}}(\mu, r, s)\right) \leq I_{\tau_{1}, \tau_{1}^{*}}\left(f^{-1}(\mu), r, s\right)$ and $C_{\tau_{1}, \tau_{1}^{*}}\left(f^{-1}\left(I_{\tau_{1}, \tau_{1}^{*}}(\mu, r, s)\right), r, s\right) \leq f^{-1}(\mu)$ for each $\mu \in I^{Y}, r \in I_{0}$, and $s \in I_{1}$.

Proof. This proof is similar to the proof of Theorem 11.

Theorem 17. Let $\left(X, \tau_{1}, \tau_{1}^{*}\right)$ and $\left(Y, \tau_{2}, \tau_{2}^{*}\right)$ be dft's. A function $f:\left(X, \tau_{1}, \tau_{1}^{*}\right) \rightarrow\left(Y, \tau_{2}, \tau_{2}^{*}\right)$ is dftc if and only if $f$ is dftsc and dftpc.

Proof. Let $\mu \in I^{Y}, r \in I_{0}$, and $s \in I_{1}$ such that $\tau_{2}(\mu) \geq r$ and $\tau_{2}^{*}(\mu) \leq s$; then $f^{-1}(\mu)$ is $(r, s)$-fsco set and $(r, s)$-fpco. From Remark $10, f^{-1}(\mu)$ is $(r, s)$-fco set in $I^{X}$. Therefore, $f$ is dftc. The completion of the proof is straightforward. 
Theorem 18. Let $f:\left(X, \tau_{1}, \tau_{1}^{*}\right) \rightarrow\left(Y, \tau_{2}, \tau_{2}^{*}\right)$ be a function. Then one has the following:

(1) if $f$ is dftc function, then $f$ is dftsc;

(2) if $f$ is dftsc function, then $f$ is $d f s c$.

Proof. (1) Let $f$ be a dftc function, $\mu \in I^{Y}, r \in I_{0}$, and $s \in I_{0}$ such that $\tau_{2}(\mu) \geq r$ and $\tau_{2}(\mu) \leq s$. By hypothesis, $f^{-1}(\mu)$ is $(r, s)$-fsco set in $I^{X}$. Therefore $f$ is a dftsc function.

(2) Let $\mu \in I^{Y}, r \in I_{0}$, and $s \in I_{1}$ such that $\tau_{2}(\mu) \geq r$ and $\tau_{2}^{*}(\mu) \leq s$. By the hypothesis, $f^{-1}(\mu)$ is $(r, s)$-fsco set in $I^{X}$. Hence $f$ is a dfsc function.

The converse of the above theorem need not be true in general as shown by the following example.

Example 19. (1) Let $X=\{a, b, c\}$ and $Y=\{x, y, z\}$. Define fuzzy sets $\lambda_{1}, \lambda_{2}$, and $\mu_{1}$ as follows:

$$
\begin{gathered}
\lambda_{1}(a)=0.4, \quad \lambda_{1}(b)=0.3, \quad \lambda_{1}(c)=0.2, \\
\lambda_{2}(a)=\mu_{1}(x)=0.6, \quad \lambda_{2}(b)=\mu_{1}(y)=0.7, \\
\lambda_{2}(c)=\mu_{1}(z)=0.8 .
\end{gathered}
$$

Let $\left(\tau_{1}, \tau_{1}^{*}\right)$ and $\left(\tau_{2}, \tau_{2}^{*}\right)$ be defined as follows:

$$
\begin{aligned}
& \tau_{1}(\lambda)= \begin{cases}1, & \text { if } \lambda=\underline{0} \text { or } \underline{1} ; \\
\frac{1}{2}, & \text { if } \lambda=\lambda_{1} ; \\
0, & \text { otherwise, }\end{cases} \\
& \tau_{1}^{*}(\lambda)= \begin{cases}0, & \text { if } \lambda=\underline{0} \text { or } \underline{1} ; \\
\frac{1}{2}, & \text { if } \lambda=\lambda_{1} ; \\
1, & \text { otherwise, }\end{cases} \\
& \tau_{2}(\mu)= \begin{cases}1, & \text { if } \mu=\underline{0} \text { or } \underline{1} ; \\
\frac{1}{2}, & \text { if } \mu=\mu_{1} ; \\
0, & \text { otherwise, }\end{cases} \\
& \tau_{2}^{*}(\mu)= \begin{cases}0, & \text { if } \mu=\underline{0} \text { or } \underline{1} ; \\
\frac{1}{2}, & \text { if } \mu=\mu_{1} ; \\
1, & \text { otherwise. }\end{cases}
\end{aligned}
$$
by

Then the function $f:\left(X, \tau_{1}, \tau_{1}^{*}\right) \rightarrow\left(Y, \tau_{2}, \tau_{2}^{*}\right)$ is defined

$$
f(a)=x, \quad f(b)=y, \quad f(c)=z .
$$

Since $\mu_{1}$ is an $(1 / 2,1 / 2)$-fo set and $f^{-1}\left(\mu_{1}\right)=\lambda_{2}$ is an $(1 / 2,1 / 2)$-fsco set not $(1 / 2,1 / 2)$-fco set, then $f$ is dftsc but not dftc.

(2) in (1) define $\lambda_{1}$ and $\mu_{1}$ as follows:

$$
\begin{array}{lll}
\lambda_{1}(a)=0.2, & \lambda_{1}(b)=0.3, & \lambda_{1}(c)=0.2, \\
\mu_{1}(x)=0.2, & \mu_{1}(y)=0.4, & \mu_{1}(z)=0.2 .
\end{array}
$$

So $f^{-1}\left(\mu_{1}\right)=\lambda_{1}$ is $(1 / 2,1 / 2)$-fso set in $\left(X, \tau_{1}, \tau_{1}^{*}\right)$ and not $(1 / 2,1 / 2)$-fsco set; that is, $f$ is not dftsc.
Definition 20. Let $f:\left(X, \tau_{1}, \tau_{1}^{*}\right) \rightarrow\left(Y, \tau_{2}, \tau_{2}^{*}\right)$ be a function between dfts's $\left(X, \tau_{1}, \tau_{1}^{*}\right)$ and $\left(Y, \tau_{2}, \tau_{2}^{*}\right)$. Then $f$ is called:

(1) double fuzzy irresolute (dfir, for short) if $f^{-1}(\mu)$ is $(r, s)$-fso set, for each $(r, s)$-fso set $\mu \in I^{Y}, r \in I_{0}$, and $s \in I_{1}$.

(2) double fuzzy semi-irresolute (dfsir, for short) if $f^{-1}(\mu)$ is $(r, s)$-fsco, for each $(r, s)$-fsco set $\mu \in I^{Y}$, $r \in I_{0}$, and $s \in I_{1}$.

Theorem 21. If a function $f:\left(X, \tau_{1}, \tau_{1}^{*}\right) \rightarrow\left(Y, \tau_{2}, \tau_{2}^{*}\right)$ is a $d$ sir function and $g:\left(Y, \tau_{2}, \tau_{2}^{*}\right) \rightarrow\left(Z, \tau_{3}, \tau_{3}^{*}\right)$ is dftc (dftsc) function, then $g \circ f:\left(X, \tau_{1}, \tau_{1}^{*}\right) \rightarrow\left(Z, \tau_{3}, \tau_{3}^{*}\right)$ is dftsc function.

Proof. Let $\mu \in I^{Z}, r \in I_{0}$, and $s \in I_{1}$ such that $\tau_{3}(\mu) \geq$ $r$ and $\tau_{3}^{*}(\mu) \leq s$. Since $g$ is a dftc (dftsc, resp.) function, $g^{-1}(\mu)$ is $(r, s)$-fco $\left((r, s)\right.$-fsco, resp.) set in $I^{Y}$. Also, since $f$ is a dfsir function, $f^{-1}\left(g^{-1}(\mu)\right)$ is $(r, s)$-fsco set in $I^{X}$. Since $(g \circ f)^{-1}(\mu)=f^{-1}\left(g^{-1}\right)(\mu),(g \circ f)^{-1}(\mu)$ is $(r, s)$-fsco set in $I^{X}$. Therefore, $g \circ f$ is a dftsc function.

\section{Semitotally Continuous Functions in Double Fuzzy Topological Spaces}

Now, we introduce the concept of semitotally continuous function which is stronger than totally continuous function in double fuzzy topological spaces, and then we investigate some characteristic properties.

Definition 22. Let $f:\left(X, \tau_{1}, \tau_{1}^{*}\right) \rightarrow\left(Y, \tau_{2}, \tau_{2}^{*}\right)$ be a function between dfts's $\left(X, \tau_{1}, \tau_{1}^{*}\right)$ and $\left(Y, \tau_{2}, \tau_{2}^{*}\right)$; then $f$ is called double fuzzy semitotally continuous function (briefly, dfstc) if $f^{-1}(\mu)$ is $(r, s)$-fco, for each $(r, s)$-fso set $\mu \in I^{Y}, r \in I_{0}$, and $s \in I_{1}$.

Theorem 23. Let $f:\left(X, \tau_{1}, \tau_{1}^{*}\right) \rightarrow\left(Y, \tau_{2}, \tau_{2}^{*}\right)$ be a function between $d f t s^{\prime} s\left(X, \tau_{1}, \tau_{1}^{*}\right)$ and $\left(Y, \tau_{2}, \tau_{2}^{*}\right)$. Then the following are equivalent:

(1) $f$ is a dfstc function,

(2) for each $x_{t} \in I^{X}$ and each $(r, s)$-fso set $\mu \in I^{Y}, r \in I_{0}$, $s \in I_{1}$, and $f\left(x_{t}\right) \leq \mu$, there exists an $(r, s)$-fco set $\lambda \in$ $I^{X}$ such that $x_{t} \in \lambda$ and $f(\lambda) \leq \mu$.

Proof. $(1) \Rightarrow(2)$ : Suppose $f:\left(X, \tau_{1}, \tau_{1}^{*}\right) \rightarrow\left(Y, \tau_{2}, \tau_{2}^{*}\right)$ is dfstc and $\mu$ is any $(r, s)$-fso set in $I^{Y}$ containing $f\left(x_{t}\right), r \in I_{0}$, and $s \in I_{1}$ so that $x_{t} \in f^{-1}(\mu)$. Take $\lambda=f^{-1}(\mu)$; then $\lambda$ is an $(r, s)$-fco set in $I^{X}$ and $x_{t} \in \lambda$, since $f$ is dfstc and $f^{-1}(\mu)$ is an $(r, s)$-fco in $I^{X}$. Also

$$
f(\lambda)=f\left(f^{-1}(\mu)\right) \leq \mu .
$$

This implies $f(\lambda) \leq \mu$.

$(2) \Rightarrow(1)$ : Suppose $\mu$ is an $(r, s)$-fso set in $I^{Y}, r \in I_{0}$, and $s \in I_{1}$ and let $x_{t} \in f^{-1}(\mu)$ be any fuzzy point; then $f\left(x_{t}\right) \leq$ $\mu$. Therefore, by hypothesis, there is an $(r, s)$-fco set $f\left(\lambda x_{t}\right)$ containing $x_{t}$ such that $f\left(x_{t}\right) \leq \mu$, so

$$
x_{t} \in \lambda x_{t} \leq f^{-1}(\mu)
$$


which implies that $f^{-1}(\mu)$ is an $(r, s)$-fco of $x_{t}$ and $\tau\left(f^{-1}(\mu)\right) \geq$ $r$ and $\tau^{*}\left(f^{-1}(\mu)\right) \leq s$ and $x_{t} q f^{-1}(\mu)$. Hence it is an $(r, s)$-fco in $I^{X}$. Therefore, $f$ is dfstc function.

Theorem 24. Let $f:\left(X, \tau_{1}, \tau_{1}^{*}\right) \rightarrow\left(Y, \tau_{2}, \tau_{2}^{*}\right)$ be a function. Then the following are equivalent:

(1) $f$ is a dfstc function,

(2) $f^{-1}(\mu)$ is an $(r, s)$-fco set of $I^{X}$ for each $(r, s)-f s c$ set $\mu \epsilon$ $I^{Y}, r \in I_{0}$, and $s \in I_{1}$,

(3)

$$
\begin{gathered}
C_{\tau_{1}, \tau_{1}^{*}}\left(f^{-1}\left(C_{\tau_{2}, \tau_{2}^{*}}\left(I_{\tau_{2}, \tau_{2}^{*}}(\mu, r, s), r, s\right)\right), r, s\right) \\
\leq f^{-1}\left(C_{\tau_{2}, \tau_{2}^{*}}\left(I_{\tau_{2}, \tau_{2}^{*}}(\mu, r, s), r, s\right)\right), \\
f^{-1}(\mu) \leq I_{\tau_{1}, \tau_{1}^{*}}\left(f^{-1}\left(I_{\tau_{2}, \tau_{2}^{*}}\left(C_{\tau_{2}, \tau_{2}^{*}}(\mu, r, s), r, s\right)\right), r, s\right)
\end{gathered}
$$

for each $(r, s)$-fso set $\mu \in I^{Y}, r \in I_{0}$, and $s \in I_{1}$,

(4)

$$
\begin{aligned}
& f^{-1}\left(I_{\tau_{2}, \tau_{2}^{*}}\left(C_{\tau_{2}, \tau_{2}^{*}}(\mu, r, s), r, s\right)\right) \\
& \quad \leq I_{\tau_{1}, \tau_{1}^{*}}\left(f^{-1}\left(I_{\tau_{2}, \tau_{2}^{*}}\left(C_{\tau_{2}, \tau_{2}^{*}}(\mu, r, s), r, s\right)\right), r, s\right), \\
& C_{\tau_{1}, \tau_{1}^{*}}\left(f^{-1}\left(C_{\tau_{2}, \tau_{2}^{*}}\left(I_{\tau_{2}, \tau_{2}^{*}}(\mu, r, s), r, s\right)\right), r, s\right) \leq f^{-1}(\mu)
\end{aligned}
$$

for each $(r, s)-f s c$ set $\mu \in I^{Y}, r \in I_{0}$, and $s \in I_{1}$.

Proof. (1) $\Rightarrow$ (2): Let $\mu \in I^{Y}$ be $(r, s)$-fsc set, $r \in I_{0}, s \in I_{1}$. Then $\underline{1}-\mu$ is $(r, s)$-fso set. By definition, $\underline{1}-f^{-1}(\mu)=f^{-1}(\underline{1}-\mu)$ is $(r, s)$-fco set in $I^{X}$. This implies that $f^{-1}(\mu)$ is $(r, s)$-fco set.

(2) $\Rightarrow$ (3): Let $\lambda \in I^{Y}$ be an $(r, s)$-fsc set, $r \in I_{0}, s \in I_{1}$; then $\underline{1}-\lambda \leq I_{\tau_{2}, \tau_{2}^{*}}\left(C_{\tau_{2}, \tau_{2}^{*}}(\underline{1}-\lambda, r, s), r, s\right)$. Since $f^{-1}(\lambda)$ is an $(r, s)$-fco set in $I^{X}$ hence $1-f^{-1}(\lambda)$ is an $(r, s)$-fso set. Take $\underline{1}-\lambda=\mu$; then by using $(\overline{3})$,

$$
\begin{aligned}
f^{-1}(\mu) & \leq f^{-1}\left(I_{\tau_{2}, \tau_{2}^{*}}\left(C_{\tau_{2}, \tau_{2}^{*}}(\mu, r, s), r, s\right)\right) \\
& \leq I_{\tau_{1}, \tau_{1}^{*}}\left(f^{-1}\left(I_{\tau_{2}, \tau_{2}^{*}}\left(C_{\tau_{2}, \tau_{2}^{*}}(\mu, r, s), r, s\right)\right), r, s\right) .
\end{aligned}
$$

(3) $\Rightarrow(4)$ : Let $\mu \in I^{Y}$ be an $(r, s)$-fsc set, $r \in I_{0}, s \in I_{1}$; then $\underline{1}-\mu$ is an $(r, s)$-fso and

$$
f^{-1}(\underline{1}-\mu) \leq f^{-1}\left(I_{\tau_{2}, \tau_{2}^{*}}\left(C_{\tau_{2}, \tau_{2}^{*}}(\underline{1}-\mu, r, s), r, s\right)\right) ;
$$

that is,

$$
f^{-1}(\mu) \geq f^{-1}\left(C_{\tau_{2}, \tau_{2}^{*}}\left(I_{\tau_{2}, \tau_{2}^{*}}(\mu, r, s), r, s\right)\right) .
$$

But $f^{-1}(\mu)$ is $(r, s)$-fsc set; then

$$
f^{-1}(\mu) \geq C_{\tau_{1}, \tau_{1}^{*}}\left(f^{-1}\left(C_{\tau_{2}, \tau_{2}^{*}}\left(I_{\tau_{2}, \tau_{2}^{*}}(\mu, r, s), r, s\right)\right), r, s\right) .
$$

(4) $\Rightarrow$ (1): Let $\mu \in I^{Y}$ be an $(r, s)$-fsc set, $r \in I_{0}, s \in I_{1}$; then

$$
f^{-1}(\mu) \geq C_{\tau_{1}, \tau_{1}^{*}}\left(f^{-1}\left(C_{\tau_{2}, \tau_{2}^{*}}\left(I_{\tau_{2}, \tau_{2}^{*}}(\mu, r, s), r, s\right)\right), r, s\right) .
$$

But $C_{\tau_{2}, \tau_{2}^{*}}(\mu, r, s)=\underline{1}-I_{\tau_{2}, \tau_{2}^{*}}(\underline{1}-\mu, r, s)$, and $\underline{1}-f^{-1}(\mu)=$ $f^{-1}(\underline{1}-\mu)$ is $(r, s)$-fco set. Thus, inverse image of every $(r, s)$ fso set is $(r, s)$-fco in $I^{X}$. Therefore, $f$ is dfstc function.

Theorem 25. Every dfstc function is a dftc function.

Proof. Suppose $f:\left(X, \tau_{1}, \tau_{1}^{*}\right) \rightarrow\left(Y, \tau_{2}, \tau_{2}^{*}\right)$ is a dfstc function and $\mu \in I^{Y}, r \in I_{0}$, and $s \in I_{1}$ such that $\tau_{2}(\mu) \geq r$ and $\tau_{2}^{*}(\mu) \leq s$. Since $\mu$ is $(r, s)$-fso set in $I^{Y}$ and $f$ is dfstc function, it follows that $f^{-1}(\mu)$ is $(r, s)$-fco in $I^{X}$. Thus inverse image of every $(r, s)$-fso set in $I^{Y}$ is $(r, s)$-fco in $I^{X}$. Therefore $f$ is dftc function.

The converse of the theorem need not be true in general as shown by the following example.

Example 26. See Example 19 (1) with $\mu_{1}$ and take $\lambda_{1}(a)=$ $\lambda_{1}(b)=\lambda_{1}(c)=0.5$. Clearly $f$ is dftc, but not dfstc.

Theorem 27. Every dfstc function is a dftsc function.

Proof. Suppose $f:\left(X, \tau_{1}, \tau_{1}^{*}\right) \rightarrow\left(Y, \tau_{2}, \tau_{2}^{*}\right)$ is a dfstc function, $\mu \in I^{Y}, r \in I_{0}$, and $s \in I_{1}$ such that $\tau_{2}(\mu) \geq r$ and $\tau_{2}^{*}(\mu) \leq s$. Since $\mu$ is $(r, s)$-fso in $I^{Y}, f$ is dfstc function. It follows that $f^{-1}(\mu)$ is $(r, s)$-fco set and hence $(r, s)$-fsco in $I^{X}$. Thus $f$ is dftsc.

The converse of the above need not be true as shown by the following example.

Example 28. Let $X=\{a, b, c\}$ and $Y=\{x, y, z\}$ define $\lambda_{1}$ and $\mu_{1}$ as follows:

$$
\begin{aligned}
& \lambda_{1}(a)=0.4, \quad \lambda_{1}(b)=0.3, \quad \lambda_{1}(c)=0.2, \\
& \mu_{1}(x)=0.3, \quad \mu_{1}(y)=0.4, \quad \mu_{1}(z)=0.5 .
\end{aligned}
$$

Define $\left(\tau_{1}, \tau_{1}^{*}\right)$ and $\left(\tau_{2}, \tau_{2}^{*}\right)$ on $X$ as follows:

$$
\tau_{1}(\lambda)= \begin{cases}1, & \text { if } \lambda=\underline{0} \text { or } \underline{1} ; \\ \frac{1}{2}, & \text { if } \lambda=\lambda_{1} ; \\ 0, & \text { otherwise, }\end{cases}
$$

$$
\tau_{1}^{*}(\lambda)= \begin{cases}0, & \text { if } \lambda=\underline{0} \text { or } \underline{1} ; \\ \frac{1}{2}, & \text { if } \lambda=\lambda_{1} ; \\ 1, & \text { otherwise, }\end{cases}
$$




$$
\begin{aligned}
& \tau_{2}(\mu)= \begin{cases}1, & \text { if } \mu=\underline{0} \text { or } \underline{1} ; \\
\frac{1}{2}, & \text { if } \mu=\mu_{1} ; \\
0, & \text { otherwise, }\end{cases} \\
& \tau_{2}^{*}(\mu)= \begin{cases}0, & \text { if } \mu=\underline{0} \text { or } \underline{1} ; \\
\frac{1}{2}, & \text { if } \mu=\mu_{1} ; \\
1, & \text { otherwise. }\end{cases}
\end{aligned}
$$

We defined the function $f:\left(X, \tau_{1}, \tau_{1}^{*}\right) \rightarrow\left(Y, \tau_{2}, \tau_{2}^{*}\right)$ by

$$
f(a)=x, \quad f(b)=y, \quad f(c)=z .
$$

So $f^{-1}\left(\mu_{1}\right)$ is $(1 / 2,1 / 2)$-fsco set but not $(1 / 2,1 / 2)$-fco set; that is, $f$ is not dfstc function.

Theorem 29. Let $\left(X, \tau_{1}, \tau_{1}^{*}\right),\left(Y, \tau_{2}, \tau_{2}^{*}\right)$, and $\left(z, \tau_{3}, \tau_{3}^{*}\right)$ be dfts's, and let $f:\left(X, \tau_{1}, \tau_{1}^{*}\right) \rightarrow\left(Y, \tau_{2}, \tau_{2}^{*}\right), g:\left(Y, \tau_{2}, \tau_{2}^{*}\right) \rightarrow$ $\left(Z, \tau_{3}, \tau_{3}^{*}\right)$, and $g \circ f:\left(X, \tau_{1}, \tau_{1}^{*}\right) \rightarrow\left(Z, \tau_{3}, \tau_{3}^{*}\right)$ be functions. Then one has the following:

(1) if $f$ is $d f$ stc and $g$ is $d f s t c$, then $g \circ f$ is $d f s t c$;

(2) if $f$ is $d f$ stc and $g$ is $d f i r$, then $g \circ f$ is $d f s t c$;

(3) if $f$ is $d f$ stc and $g$ is $d f s c$, then $g \circ f$ is $d f t c$;

(4) if $f$ is $d$ stc and $g$ is any function, then $g \circ f$ is $d f$ stc if and only if $g$ is dfir.

Proof. (1) It is clear.

(2) Let $\mu$ be an $(r, s)$-fso set in $I^{Z}, r \in I_{0}$, and $s \in I_{1}$ and $g$ is dfir, so $g^{-1}(\mu)$ is $(r, s)$-fso set in $I^{Y}$. Also since $f$ is dfstc function, then $f^{-1}\left(g^{-1}(\mu)\right)=(g \circ f)^{-1}(\mu)$ is $(r, s)$-fco set in $I^{X}$. Hence $g \circ f$ is dfstc function.

(3) It is similar to the proof of (2).

(4) The proof follows from (2).

Conversely, let $g \circ f$ be dfstc and let $\mu$ be an $(r, s)$-fso set in $I^{Z}, r \in I_{0}$, and $s \in I_{1}$. Now, by hypothesis $g \circ f$ is dfstc; $(g \circ f)^{-1}(\mu)=f^{-1}\left(g^{-1}(\mu)\right)$ is $(r, s)$-fco in $I^{X}$. But $f$ is dfstc; then $g^{-1}(\mu)$ is $(r, s)$-fso in $I^{Y}$. Hence $g$ is dfir.

Definition 30. Let $\left(X, \tau, \tau^{*}\right)$ be a dfts. Then it is called:

(1) double fuzzy semi- $T_{0}$, if, for each pair of distinct fuzzy points in $I^{X}$, there exists an $(r, s)$-fso set containing one fuzzy point but not the other, $r \in I_{0}$ and $s \in I_{1}$.

(2) double fuzzy semi- $T_{1}$ (resp., double fuzzy clopen $T_{1}$ ) if, for each pair of distinct fuzzy points $x_{n}$ and $y_{m}$ of $I^{X}, r \in I_{0}$, and $s \in I_{1}$, there exist $(r, s)$-fso (resp., $(r, s)$ fco) sets $\lambda$ and $\mu$ containing $x_{n}$ and $y_{m}$, respectively, such that $x_{n} \in \lambda, y_{m} \notin \lambda$ and $x_{n} \notin \mu, y_{m} \in \mu$.

(3) double fuzzy semi- $T_{2}$ (resp., double fuzzy ultra- $T_{2}$ ) if, for each disjoint points $x_{n}$ and $y_{m}$ of $I^{X}$ can be separated by disjoint $(r, s)$-fso (resp., $(r, s)$-fco) sets, $r \in I_{0}$ and $s \in I_{1}$.

(4) double fuzzy ultranormal if, for each pair of nonzero disjoint $(r, s)$-fc sets can be separated by disjoint $(r, s)$ fco sets, $r \in I_{0}$ and $s \in I_{1}$.
(5) double fuzzy seminormal if, for each pair of disjoint $(r, s)$-fsc sets $\lambda$ and $\mu$ of $I^{X}$, there exist two disjoint $(r, s)$-fso sets $\alpha$ and $\beta, r \in I_{0}$ and $s \in I_{1}$ such that $\lambda \leq \alpha$ and $\mu \leq \beta$.

(6) double fuzzy s-connected if $\left(X, \tau, \tau^{*}\right)$ is not the union of two disjoint nonzero $(r, s)$-fso subsets of $I^{X}, r \in I_{0}$, and $s \in I_{1}$.

Theorem 31. Let $\left(X, \tau_{1}, \tau_{1}^{*}\right)$ and $\left(Y, \tau_{2}, \tau_{2}^{*}\right)$ be dfts's and $f$ : $\left(X, \tau_{1}, \tau_{1}^{*}\right) \rightarrow\left(Y, \tau_{2}, \tau_{2}^{*}\right)$ be a function. Then one has the following.

(1) If $f$ is $d f$ stc injection and $\left(Y, \tau_{2}, \tau_{2}^{*}\right)$ is double fuzzy semi- $T_{1}$, then $\left(X, \tau_{1}, \tau_{1}^{*}\right)$ is double fuzzy clopen- $T_{1}$.

(2) If $f$ is dfstc injection and $\left(Y, \tau_{2}, \tau_{2}^{*}\right)$ is double fuzzy semi- $T_{0}$, then $\left(X, \tau_{1}, \tau_{1}^{*}\right)$ is double fuzzy ultra- $T_{2}$.

(3) If $f$ is dfstc injection and $\left(Y, \tau_{2}, \tau_{2}^{*}\right)$ is double fuzzy semi- $T_{2}$, then $\left(X, \tau_{1}, \tau_{1}^{*}\right)$ is double fuzzy ultra- $T_{2}$.

(4) If $f$ is $d f$ stc injective double fuzzy semiopen function from a double fuzzy clopen regular space $\left(X, \tau_{1}, \tau_{1}^{*}\right)$ onto $\left(Y, \tau_{2}, \tau_{2}^{*}\right)$, then $\left(Y, \tau_{2}, \tau_{2}^{*}\right)$ is double fuzzy semiregular.

(5) If $f$ is dfstc injective double fuzzy semiopen function from a double fuzzy clopen normal space $\left(X, \tau_{1}, \tau_{1}^{*}\right)$ onto $\left(Y, \tau_{2}, \tau_{2}^{*}\right)$, then $\left(Y, \tau_{2}, \tau_{2}^{*}\right)$ is double fuzzy seminormal.

Proof. (1) Suppose fuzzy points $x_{n}$ and $y_{m}$ are in $I^{X}$ such that $x_{n} \neq y_{m}$. Since $f$ is injective, then $f\left(x_{n}\right) \neq f\left(y_{m}\right)$ in $I^{Y}$. Also $\left(Y, \tau_{2}, \tau_{2}^{*}\right)$ is double fuzzy semi- $T_{1}$ so, there exist $\lambda$ and $\mu$ which are $(r, s)$-fso sets in $I^{Y}, r \in I_{0}$, and $s \in I_{1}$ such that $f\left(x_{n}\right) \leq \lambda, f\left(y_{m}\right) \not \lambda, f\left(y_{m}\right) \leq \mu$, and $f\left(y_{m}\right) \not \lambda$; that is, $x_{n} \in f^{-1}(\lambda), y_{m} \notin f^{-1}(\lambda), y_{m} \in f^{-1}(\mu)$, and $x_{n} \notin f^{-1}(\mu)$. Since $f$ is dfstc, then $f^{-1}(\lambda)$ and $f^{-1}(\mu)$ are $(r, s)$-fco subsets of $I^{X}$. That is, $\left(X, \tau_{1}, \tau_{1}^{*}\right)$ is double fuzzy clopen $T_{1}$.

(2) Suppose fuzzy points $x_{n}$ and $y_{m} \in I^{X}$ such that $x_{n} \neq y_{m}$. Since $f$ is injective, then $f\left(x_{n}\right) \neq f\left(y_{m}\right)$ in $I^{Y}$. Also $\left(Y, \tau_{2}, \tau_{2}^{*}\right)$ is double fuzzy semi- $T_{0}$ so there exists an $(r, s)$-fso set $\lambda, r \in I_{0}, s \in I_{1}$ such that $f\left(x_{n}\right) \leq \lambda$ but $f\left(y_{m}\right) \not \lambda$; that is, $x_{n} \in f^{-1}(\lambda), y_{m} \notin f^{-1}(\lambda)$. But $f$ is dfstc; $f^{-1}(\lambda)$ is $(r, s)$ fco set in $I^{X}$. This implies that every pair of distinct points of $I^{X}$ can be separated by disjoint $(r, s)$-fco sets in $I^{X}$. Therefore $\left(X, \tau_{1}, \tau_{1}^{*}\right)$ is double fuzzy ultra- $T_{2}$.

(3) Suppose fuzzy points $x_{n}$ and $y_{m} \in I^{X}$ such that $x_{n} \neq y_{m}$. Since $f$ is injective, then $f\left(x_{n}\right) \neq f\left(y_{m}\right)$ in $I^{Y}$. But $\left(Y, \tau_{2}, \tau_{2}^{*}\right)$ is double fuzzy semi- $T_{2}$ so there exist $\lambda$ and $\mu$ which are $(r, s)$-fso sets in $I^{Y}, r \in I_{0}$, and $s \in I_{1}$ such that $f\left(x_{n}\right) \leq \lambda$, $f\left(y_{m}\right) \leq \mu$, and $\lambda \wedge \mu=0$; that is, $x_{n} \in f^{-1}(\lambda)$ and $y_{m} \in f^{-1}(\mu)$. But $f$ is dfstc; $f^{-1}(\lambda)$ and $f^{-1}(\mu)$ are $(r, s)$ fco sets in $I^{X}$ such that $f^{-1}(\lambda) \wedge f^{-1}(\mu)=f^{-1}(\lambda \wedge \mu)=\underline{0}$. Therefore $\left(X, \tau_{1}, \tau_{1}^{*}\right)$ is double fuzzy ultra- $T_{2}$.

(4) Suppose $\beta$ is an $(r, s)$-fsc set in $I^{Y}$ and $y_{n} \notin \beta$. Assume $y_{n}=f\left(x_{t}\right)$ and $\alpha=f^{-1}(\beta)$. Since $f$ is dftsc function, then $f^{-1}(\beta)$ is an $(r, s)$-fco set in $I^{X}$ such that $x_{t} \notin \alpha$. But $\left(X, \tau_{1}, \tau_{1}^{*}\right)$ 
is double fuzzy clopen regular; then there exist disjoint $(r, s)$ fo sets $\lambda$ and $\mu$ such that $\alpha \leq \lambda$ and $x_{t} \in \mu$, which implies

$$
\begin{gathered}
\beta=f(\alpha) \leq f(\lambda), \\
y_{n}=f\left(x_{t}\right) \leq f(\mu) .
\end{gathered}
$$

Also, by hypothesis $f$ is injective double fuzzy semiopen; then $f(\lambda)$ and $f(\mu)$ are $(r, s)$-fso sets such that

$$
f(\lambda) \wedge f(\mu)=f(\lambda \wedge \mu)=f(\underline{0})=\underline{0} .
$$

Therefore $\left(Y, \tau_{2}, \tau_{2}^{*}\right)$ is double fuzzy semiregular.

(5) Assume that $\beta_{1}$ and $\beta_{2}$ are any two disjoint $(r, s)$-fsc sets in $\left(I^{Y}\right)$. Take $\lambda=f^{-1}\left(\beta_{1}\right)$ and $\mu=f^{-1}\left(\beta_{2}\right)$. Since $f$ is dfstc injective, then $f^{-1}\left(\beta_{1}\right)$ and $f^{-1}\left(\beta_{2}\right)$ are $(r, s)$-fco subsets of $I^{X}$ such that

$$
\lambda \wedge \mu=f^{-1}\left(\beta_{1}\right) \wedge f^{-1}\left(\beta_{2}\right)=f^{-1}\left(\beta_{1} \wedge \beta_{2}\right)=f^{-1}(\underline{0})=\underline{0} .
$$

$\operatorname{But}\left(X, \tau_{1}, \tau_{1}^{*}\right)$ is double fuzzy clopen normal; then there exist disjoint $(r, s)$-fo sets $\delta_{1}$ and $\delta_{2}$ such that $\lambda \leq \delta_{1}$ and $\mu \leq \delta_{2}$ which implies

$$
\begin{aligned}
& \beta_{1}=f(\lambda) \leq f\left(\delta_{1}\right), \\
& \beta_{2}=f(\mu) \leq f\left(\delta_{2}\right) .
\end{aligned}
$$

Also, since $f$ is injective double fuzzy semiopen, $f\left(\delta_{1}\right)$ and $f\left(\delta_{2}\right)$ are disjoint $(r, s)$-fso sets. Therefore $\left(Y, \tau_{2}, \tau_{2}^{*}\right)$ is double fuzzy seminormal.

Theorem 32. Let $\left(X, \tau_{1}, \tau_{1}^{*}\right)$ and $\left(Y, \tau_{2}, \tau_{2}^{*}\right)$ be dfts's and let $f:\left(X, \tau_{1}, \tau_{1}^{*}\right) \rightarrow\left(Y, \tau_{2}, \tau_{2}^{*}\right)$ be a function. Then one has the following.

(1) If $f$ is dfstc, double fuzzy closed injection and $\left(Y, \tau_{2}, \tau_{2}^{*}\right)$ is double fuzzy s-normal, then $\left(X, \tau_{1}, \tau_{1}^{*}\right)$ is double fuzzy ultranormal.

(2) If $f$ is dfstc, double fuzzy semiclosed injection and $\left(Y, \tau_{2}, \tau_{2}^{*}\right)$ is double fuzzy s-regular, then $\left(X, \tau_{1}, \tau_{1}^{*}\right)$ is double fuzzy ultraregular.

Proof. (1) Suppose $\mu_{1}, \mu_{2}$ are disjoint $(r, s)$-fc subsets in $I^{X}$, $r \in I_{0}$, and $s \in I_{1}$. Since $f$ is double fuzzy closed and injective, then $\tau_{2}\left(1-f\left(\mu_{1}\right)\right) \geq \tau_{1}\left(\underline{1}-\mu_{1}\right), \tau_{2}^{*}\left(1-f\left(\mu_{1}\right)\right) \leq \tau_{1}^{*}\left(\underline{1}-\mu_{1}\right)$, $\tau_{2}\left(\underline{1}-f\left(\mu_{2}\right)\right) \geq \tau_{1}\left(\underline{1}-\mu_{2}\right)$, and $\tau_{2}^{*}\left(\underline{1}-f\left(\mu_{2}\right)\right) \leq \tau_{1}^{*}\left(\underline{1}-\mu_{2}\right)$, for each $\mu_{1}, \mu_{2} \in I^{\bar{X}}, r \in I_{0}$, and $s \in I_{1}$ such that $f\left(\mu_{1}\right)$ and $f\left(\mu_{2}\right)$ are disjoint $(r, s)$-fc subsets in $I^{Y}$, so $f\left(\mu_{1}\right)$ and $f\left(\mu_{2}\right)$ are separated by disjoint $(r, s)$-fso sets $\lambda_{1}, \lambda_{2}$, respectively, because $\left(Y, \tau_{2}, \tau_{2}^{*}\right)$ is double fuzzy s-normal space; then $\mu_{1} \leq$ $f^{-1}\left(\lambda_{1}\right)$ and $\mu_{2} \leq f^{-1}\left(\lambda_{2}\right)$. Also $f$ is dfstc, and $f^{-1}\left(\lambda_{1}\right)$ and $f^{-1}\left(\lambda_{2}\right)$ are $(r, s)$-fco sets in $I^{X}$ such that

$$
f^{-1}\left(\lambda_{1}\right) \wedge f^{-1}\left(\lambda_{2}\right)=f^{-1}\left(\lambda_{1} \wedge \lambda_{2}\right)=\underline{0} .
$$

Therefore $\left(X, \tau_{1}, \tau_{1}^{*}\right)$ is double fuzzy ultranormal.

(2) Assume that an $(r, s)$-fc set $\alpha$ does not contain fuzzy point $x_{t}$ and let $f$ be a double fuzzy semiclosed function; then $f(\alpha)$ is an $(r, s)$-fsc set in $I^{Y}$ such that $f(\alpha)$ does not contain $f\left(x_{t}\right)$. Since $\left(Y, \tau_{2}, \tau_{2}^{*}\right)$ is double fuzzy semiregular, then there exist disjoint $(r, s)$-fso sets $\lambda$ and $\mu$ such that $f\left(x_{t}\right) \leq \lambda$ and $f(\alpha) \leq \mu$; that is $x_{t} \in f^{-1}(\lambda)$ and $\alpha \leq f^{-1}(\mu)$. Since $f$ is dfstc function, then $f^{-1}(\lambda)$ and $f^{-1}(\mu)$ are $(r, s)$-fco sets in $I^{X}$. But $f$ is injective; then

$$
f^{-1}(\lambda) \wedge f^{-1}(\mu)=f^{-1}(\lambda \wedge \mu)=f^{-1}(\underline{0})=\underline{0} .
$$

Therefore, $\left(X, \tau_{1}, \tau_{1}^{*}\right)$ is double fuzzy ultraregular.

Theorem 33. If $f:\left(X, \tau_{1}, \tau_{1}^{*}\right) \rightarrow\left(Y, \tau_{2}, \tau_{2}^{*}\right)$ is dfstc surjection and $\left(X, \tau_{1}, \tau_{1}^{*}\right)$ is double fuzzy connected, then $\left(Y, \tau_{2}, \tau_{2}^{*}\right)$ is double fuzzy s-connected.

Proof. Assume $\left(Y, \tau_{2}, \tau_{2}^{*}\right)$ is not double fuzzy s-connected and $\lambda$ and $\mu$ are $(r, s)$-fso sets in $I^{Y}, r \in I_{0}$, and $s \in I_{1}$ such that $\lambda \wedge \mu=\underline{1}$ and $\lambda \wedge \mu=\underline{0}$. Since $f$ is dfstc,

$$
f^{-1}(\lambda \wedge \mu)=f^{-1}(\lambda) \wedge f^{-1}(\mu)=f^{-1}(\underline{1})=\underline{1} .
$$

Also $f^{-1}(\lambda)$ and $f^{-1}(\mu)$ are nonzero $(r, s)$-fco sets in $I^{X}$ such that

$$
f^{-1}(\lambda) \wedge f^{-1}(\mu)=f^{-1}(\lambda \wedge \mu)=\underline{0} .
$$

Then $\left(X, \tau_{1}, \tau_{1}^{*}\right)$ is not double fuzzy connected, so it is a contradiction. Hence $\left(Y, \tau_{2}, \tau_{2}^{*}\right)$ is double fuzzy s-connected.

\section{Conclusion}

In this paper, we introduced and characterized the notions of totally continuous functions, totally semicontinuous functions, and semitotally continuous functions in double fuzzy topological spaces. The relationship with other kinds of functions is studied. We could know that double fuzzy topological spaces are a generalization of some other kinds of topological spaces; therefore, our results can be considered as a generalization of the same results in other kinds of topological spaces. Also, it is possible to study this topic for a completely distributive DeMorgan algebra.

\section{Conflict of Interests}

The authors declare that there is no conflict of interests regarding the publication of this paper.

\section{Acknowledgments}

The authors would like to acknowledge the financial support received from Universiti Kebangsaan Malaysia under the research Grant GUP-2013-040. The authors also wish to gratefully acknowledge all those who have generously given their time to referee our paper.

\section{References}

[1] L. A. Zadeh, "Fuzzy sets," Information and Computation, vol. 8, pp. 338-353, 1965. 
[2] C. L. Chang, "Fuzzy topological spaces," Journal of Mathematical Analysis and Applications, vol. 24, pp. 39-90, 1968.

[3] D. Coker, "An introduction to fuzzy subspaces in intuitionistic fuzzy topological spaces," Journal of Fuzzy Mathematics, vol. 4, no. 4, pp. 749-764, 1996.

[4] D. Çoker, "An introduction to intuitionistic fuzzy topological spaces," Fuzzy Sets and Systems, vol. 88, no. 1, pp. 81-89, 1997.

[5] M. Demirci and D. Çoker, "An introduction to intuitionistic fuzzy topological spaces in Sostak's sense," Busefal, vol. 67, pp. 61-66, 1996.

[6] T. K. Mondal and S. K. Samanta, "On intuitionistic gradation of openness," Fuzzy Sets and Systems, vol. 131, no. 3, pp. 323-336, 2002.

[7] J. Gutiérrez García and S. E. Rodabaugh, "Order-theoretic, topological, categorical redundanci es of interval-valued sets, grey sets, vague sets, interval-valued; intuitionistic sets, intuitionistic fuzzy sets and topologies," Fuzzy Sets and Systems, vol. 156, no. 3, pp. 445-484, 2005.

[8] R. C. Jain, The role of regularly open sets in general topology [Ph.D. thesis], Meerut University, Meerut, India, 1980.

[9] A. Mukherjee, "Fuzzy totally continuous and totally semicontinuous functions," Fuzzy Sets and Systems, vol. 107, no. 2, pp. 227-230, 1999.

[10] A. Mani-Maran and P. Thangaraj, "Intuitionistic fuzzy totally continuous and totally semicontinuous mappings in intuitionistic fuzzy topological spaces," International Journal of Advanced Scientific and Technical Research, vol. 2, pp. 2249-2254, 2011.

[11] S.-Z. Bai, "A note on the article 'Fuzzy totally continuous and totally semi-continuous functions," Fuzzy Sets and Systems, vol. 140, no. 3, pp. 555-558, 2003.

[12] A. Ghareeb, "Normality of double fuzzy topological spaces," Applied Mathematics Letters, vol. 24, no. 4, pp. 533-540, 2011.

[13] M. A. Abd-Allah, K. El-Saady, and A. Ghareeb, "(r, s)-Fuzzy Fopen sets and (r, s)-fuzzy F-closed spaces," Chaos, Solitons \& Fractals, vol. 42, no. 2, pp. 649-656, 2009.

[14] A. M. Zahran, M. A. Abd-Allah, and A. E. G. Abd El-Rahman, "Fuzzy weakly preopen (preclosed) function in Kubiak-Šostak fuzzy topological spaces," Chaos, Solitons and Fractals, vol. 39, no. 3, pp. 1158-1168, 2009.

[15] A. Ghareeb, "Weak forms of continuity in I-double gradation fuzzy to pological spaces," Springerplus, vol. 19, no. 1, 9 pages, 2012.

[16] S. E. Abbas and E. El-Sanousy, "Several types of double fuzzy semiclosed sets," The Journal of Fuzzy Mathematics, vol. 20, no. 1, pp. 89-102, 2012. 

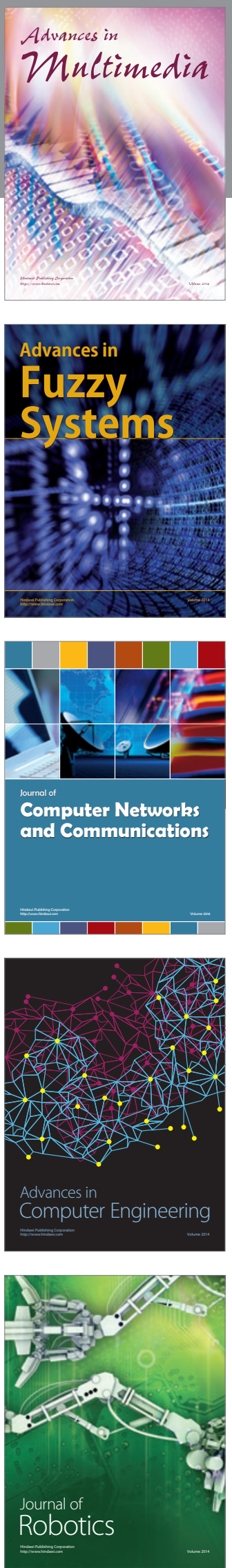

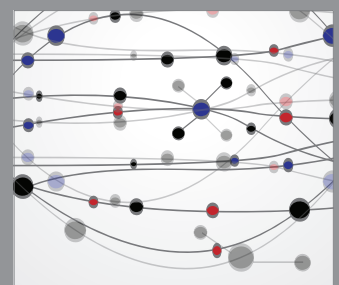

The Scientific World Journal
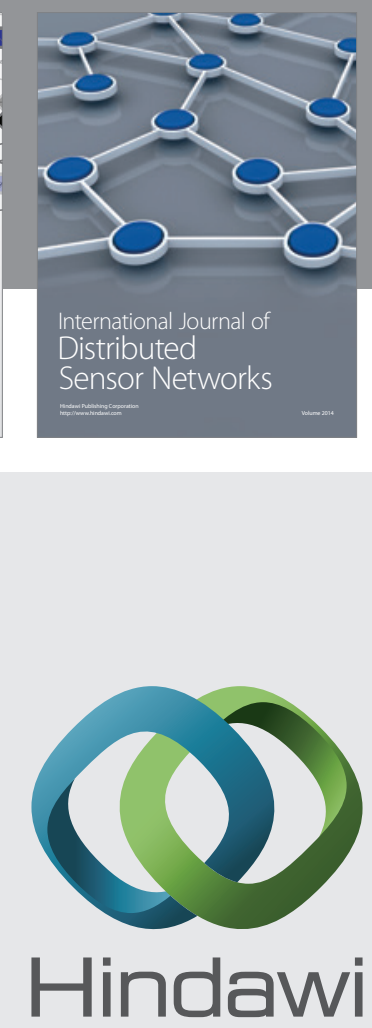

Submit your manuscripts at

http://www.hindawi.com
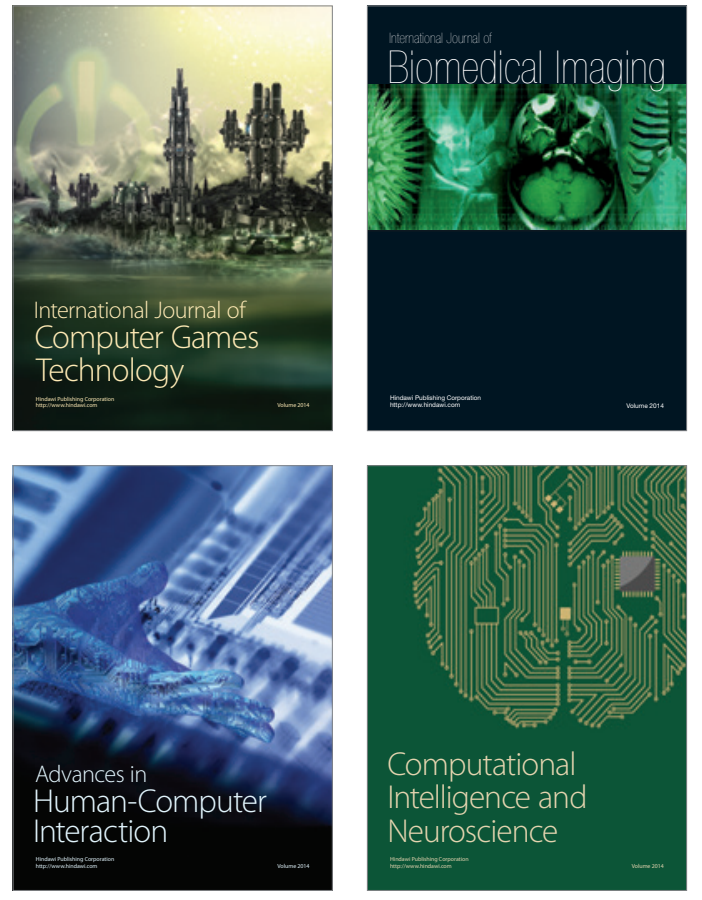
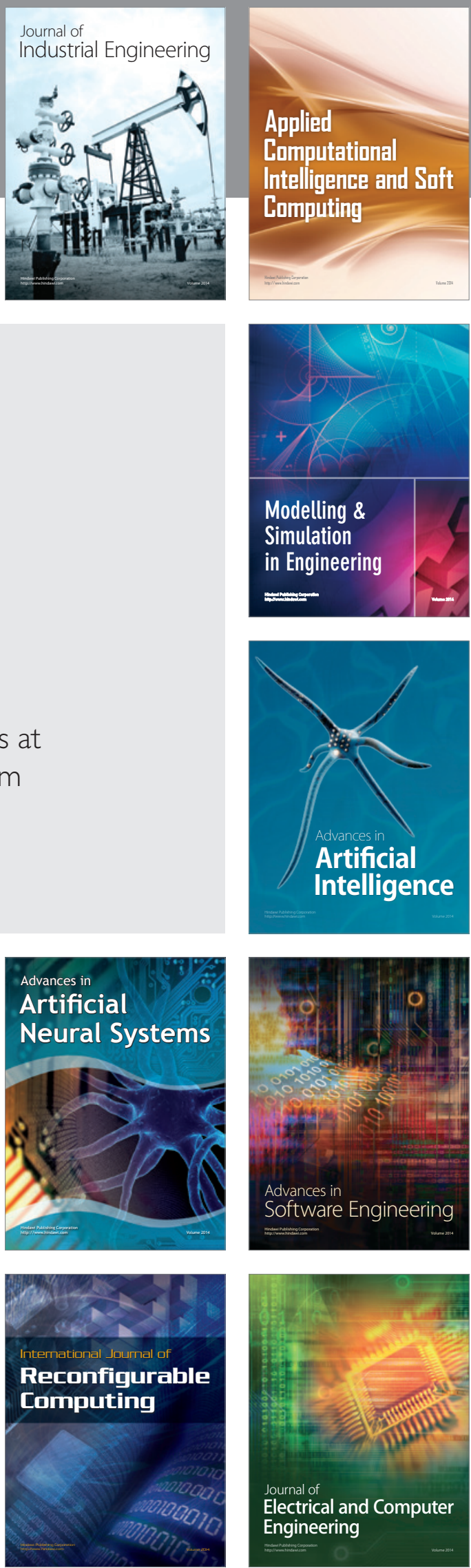